\title{
IdeAs
}

Idées d'Amériques

$17 \mid 2021$

Villes et culture dans les Amériques

\section{Marcher dans les rues de Las Vegas : renouvellement des pratiques touristiques et reconfigurations spatiales des hôtels-casinos}

Walking in the streets of Las Vegas : renewal of tourists practices and hotelcasinos' spatial reconfigurations

Caminar por las calles de Las Vegas : renovación de las prácticas turísticas y reconfiguraciones espaciales de hoteles casino

Pascale Nédélec

\section{OpenEdition}

Journals

Édition électronique

URL : https://journals.openedition.org/ideas/10621

DOI : 10.4000/ideas. 10621

ISSN : $1950-5701$

Éditeur

Institut des Amériques

Référence électronique

Pascale Nédélec, «Marcher dans les rues de Las Vegas : renouvellement des pratiques touristiques et reconfigurations spatiales des hôtels-casinos », IdeAs [En ligne], 17| 2021, mis en ligne le 01 mars 2021, consulté le 04 juin 2021. URL : http://journals.openedition.org/ideas/10621 ; DOI : https:// doi.org/10.4000/ideas. 10621

Ce document a été généré automatiquement le 4 juin 2021

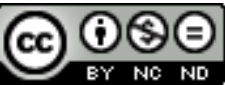

IdeAs - Idées d'Amériques est mis à disposition selon les termes de la licence Creative Commons Attribution - Pas d'Utilisation Commerciale - Pas de Modification 4.0 International. 


\title{
Marcher dans les rues de Las Vegas : renouvellement des pratiques touristiques et reconfigurations spatiales des hôtels-casinos
}

\author{
Walking in the streets of Las Vegas : renewal of tourists practices and hotel- \\ casinos' spatial reconfigurations \\ Caminar por las calles de Las Vegas : renovación de las prácticas turísticas y \\ reconfiguraciones espaciales de hoteles casino
}

Pascale Nédélec

\section{Introduction}

1 Las Vegas est l'auto-proclamée «capitale mondiale du jeu " (Gambling Capital of the World), connue mondialement pour ses hôtels-casinos et leurs tables de jeu, alignés le long du "Strip ». Ce toponyme désigne à la fois une portion, longue de six kilomètres, de l'artère routière Las Vegas Boulevard qui traverse l'aire urbaine ${ }^{1} \mathrm{du}$ nord au sud, et le quartier touristique qui a fait la renommée de la destination végasienne aux EtatsUnis et dans le reste du monde. Ce quartier polarise l'essentiel des quarante-deux millions et demi de touristes qui ont visité Las Vegas en 2019, en faisant l'une des destinations les plus visitées du pays. Son attraction réside en effet non seulement dans les jeux d'argent mais aussi dans la très grande diversité de divertissements proposés accessibles à tous les budgets.

Le Las Vegas Boulevard, et par conséquent le Strip, est une ancienne autoroute intraurbaine qui s'est métamorphosée sous l'influence des nouvelles attentes des touristes. Le quartier a en effet été conçu dans les années 1940 autour des déplacements automobiles. Pourtant, les trottoirs du Strip sont désormais les plus animés et les plus empruntés de l'aire urbaine et incarnent une part croissante de l'attractivité de Las 
Vegas aux yeux des visiteurs. Cet article étudie ainsi l'essor de la marche à pied dans le quartier touristique du Strip à Las Vegas depuis les années 1990. Il démontre la rupture nette constituée par l'affirmation de la marche à pied comme principale pratique touristique de mobilité. Cette évolution des pratiques a conduit à une réorganisation physique des hôtels-casinos qui a modifié le rapport à la rue et à l'espace public.

Cet article étudie plus particulièrement la dimension actorielle de l'évolution des pratiques touristiques de mobilité en interrogeant le rôle respectif des acteurs privés propriétaires des hôtels-casinos, des acteurs publics et des acteurs individuels que sont les touristes. Les reconfigurations spatiales engendrées par l'essor de la marche à pied sont étudiées en détail à partir de deux projets urbains récents (années 2010) : The Linq Promenade et The Park Vegas. Ces derniers confirment la rupture dans la conception même des hôtels-casinos du Strip : de l'introversion vers l'extraversion et l'extension dans le domaine public de la rue et des trottoirs. Ils sont pris en exemple pour étudier la capacité d'adaptation des hôtels-casinos aux nouvelles attentes des touristes, et plus largement pour questionner les différents positionnements marketing de ces acteurs clés de l'aménagement de l'espace végasien. Ces deux aménagements urbains offrent enfin un support de réflexion pour interroger les dynamiques de publicisation apparente et de privatisation des espaces publics des trottoirs du Strip.

Une présentation historique de la naissance du Strip met en évidence sa conception et sa structuration intrinsèque par les déplacements automobiles des années 1940 aux années 1990. A partir des années 1990, l'affirmation de la marche comme nouvelle pratique touristique constitue une rupture nette due au nouveau positionnement marketing d'acteurs phares parmi les propriétaires d'hôtels-casinos. Deux projets urbains, The Linq Promenade et The Park Vegas sont enfin étudiés pour démontrer la capacité des propriétaires d'hôtels-casinos à accompagner le renouvellement des pratiques touristiques.

\section{Le Strip : un quartier touristique calibré pour et par les touristes automobilistes}

\section{La naissance du Strip dans les années 1940}

5 Alors que la ville est officiellement fondée en 1905 (Moehring E. et M. Green, 2005) jusque dans les années 1940, City of Las Vegas demeure une simple ville étape sur des circuits touristiques à destination du barrage Hoover ou du Grand Canyon (situés respectivement à soixante kilomètres et cent trente kilomètres à vol d'oiseau au sudest), et n'arrive pas à fixer les touristes de passage. Il faut attendre avril 1941 pour que Thomas Hull, entrepreneur hôtelier californien, décide d'ouvrir un hôtel-casino, El Rancho, juste au Sud des limites municipales de City of Las Vegas (délimitée par Sahara Avenue), le long de l'autoroute 91 sur le territoire du comté de Clark (Schwartz D., 2003, Moehring E., 2000). A première vue, une telle localisation peut surprendre: l'établissement $\mathrm{El}$ Rancho est éloigné du centre-ville de City of Las Vegas (trois kilomètres au sud-ouest), situé dans ce qui peut paraitre le milieu de nulle part, entouré de vastes étendues désertiques sans intérêt, le long d'une autoroute Los Angeles / Las Vegas encore peu fréquentée. Néanmoins, c'est bien cet éloignement de la ville qui est recherché par Hull : en construisant son hôtel-casino en dehors des limites municipales, il bénéficie de terrains à bas coût, tout en évitant les prélèvements fiscaux 
municipaux, la concurrence des commerces du centre-ville et l'interférence des hommes politiques de City of Las Vegas dans ses affaires.

Figure 1 : Localisations emboîtées : du Nevada au Strip

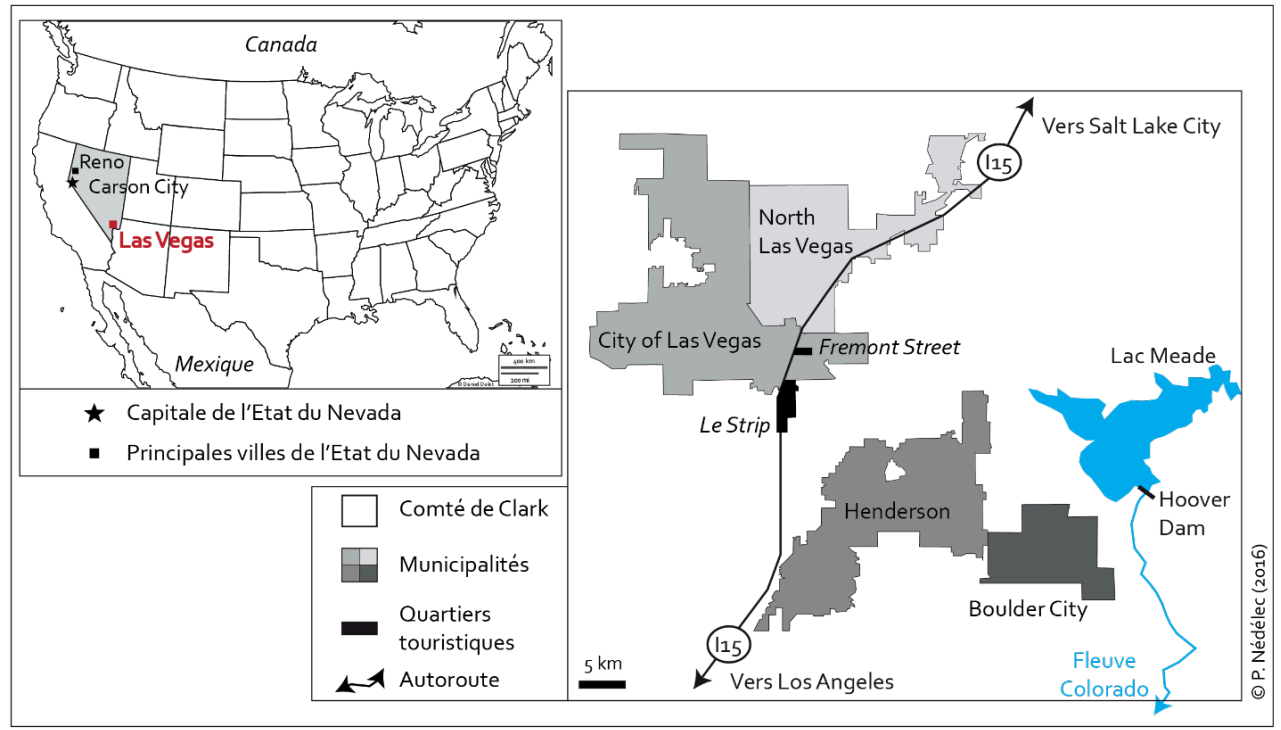

Source : P. Nédélec (2016)

6 L'isolement et l'éloignement du Nevada en général et de Las Vegas en particulier présentent également un avantage pour les touristes qui viennent s'y adonner légalement aux jeux d'argent, encore largement condamnés socialement jusque dans les années 1970-1980². Pour les clients, les casinos situés le long de l'autoroute 91 constituent un havre sécurisant pour fréquenter les tables de jeu, sans avoir à s'aventurer dans les quartiers dangereux du centre-ville, où se situent habituellement les cercles de jeux tenus par des mafieux et propices à la criminalité, et à l'écart de leur foyer, n'en menaçant par conséquent pas la tranquillité. L'historien D. Schwartz (2003) parle ainsi de « confinement du jeu » en périphérie de la nation et de la ville de City of Las Vegas. On constate donc que, dès l'origine, ce qui va devenir le Strip s'est construit dans le rejet de la ville et de ses contraintes fiscales.

7 Les années 1940-1960 fixent les nœuds de peuplement dans la région et marquent l'ébauche des axes de développement de l'aire urbaine naissante. Les établissements se multiplient le long de ce qui est encore une artère autoroutière : en vingt ans, onze nouveaux hôtels-casinos sont construits à proximité du El Rancho ${ }^{3}$. C'est également à cette époque que se fixent les toponymes en usage aujourd'hui: la portion de l'autoroute 91 traversant le bassin de Las Vegas est officiellement nommée le "Las Vegas Boulevard » en 1959 et la portion le long de laquelle sont concentrés les hôtelscasinos, soit entre Sahara Avenue au Nord et Russel Road au Sud, est surnommée le «Strip $»^{4}$.

8 Le quartier touristique du Strip s'est ainsi imposé comme une synecdoque de l'ensemble du bassin de Las Vegas. Ce n'est ni le lieu originel de peuplement de la région, ni le quartier touristique le plus ancien ${ }^{5}$, ni même un quartier de la ville de City of Las Vegas. Pourtant, le quartier incarne et résume dans les représentations collectives la mise en tourisme de Las Vegas et sa spécialisation autour des jeux d'argent dans des hôtels-casinos aux dimensions gigantesques. Il faut ainsi souligner 
l'emploi courant mais abusif du toponyme "Las Vegas" pour ne désigner que le quartier touristique du Strip. Il témoigne de la vision déformée portée sur l'aire urbaine (Nédélec P., 2017) qui est bien plus étendue spatialement, et dont l'activité économique est diversifiée grâce à sa population de 2,2 millions d'habitants en 2019.

\section{Un quartier touristique calibré autour d'une ancienne autoroute}

9 La conception des hôtels-casinos du Strip a été fondamentalement influencée par leur localisation le long d'une autoroute. Ainsi, il s'agissait pour les propriétaires de construire des établissements non seulement visibles, mais aussi suffisamment attractifs pour attirer l'œil des touristes arrivant en voiture à des vitesses relativement élevées. L'architecture du Strip est une conséquence directe de la domination des déplacements automobiles dans les pratiques touristiques, comme l'ont démontré les architectes R. Venturi, D. Scott-Brown et S. Izenour dans leur ouvrage de référence Learning from Las Vegas $(1972,1977)$. Ceci explique le caractère monumental des hôtelscasinos et le recours à des panneaux publicitaires massifs, dominant la route de plusieurs dizaines de mètres de haut. L'hôtel-casino The Sands, célèbre notamment pour les spectacles de Franck Sinatra, était par exemple annoncé par une enseigne (marquee) de dix-sept mètres de haut (soit l'équivalent d'un immeuble de six ou sept étages).

10 L'augmentation du trafic automobile dans les années 1970 fait évoluer le statut du Las Vegas Boulevard. Pour accueillir les flux croissants d'automobilistes, le Las Vegas Boulevard est déclassé en 1974, passant ainsi du statut d'autoroute à celui de boulevard urbain. En parallèle, une nouvelle autoroute, plus large, est construite à cinqcents mètres à l'ouest, juste derrière les complexes hôteliers. L'autoroute Interstate 15 (I15) maintient, voire renforce l'accessibilité de Las Vegas pour les touristes automobilistes et plusieurs échangeurs sont construits pour faciliter l'accès au Strip. L'héritage autoroutier domine encore aujourd'hui (cf. photographie 1): les hôtelscasinos et autres attractions touristiques du Strip sont construits de part et d'autre d'une voie routière de deux fois six voies, d'une largeur oscillant entre quarante et cinquante mètres en moyenne. Des aménagements paysagers ont néanmoins été ajoutés pour atténuer l'aspect autoroutier : un terre-plein central planté de palmiers sépare les deux voies de circulation. De plus, des passages piétons ont été installés à toutes les intersections pour permettre les déplacements piétonniers. 
Photographie 1 : Vue sur le Strip : héritage d'une voirie de calibre autoroutier

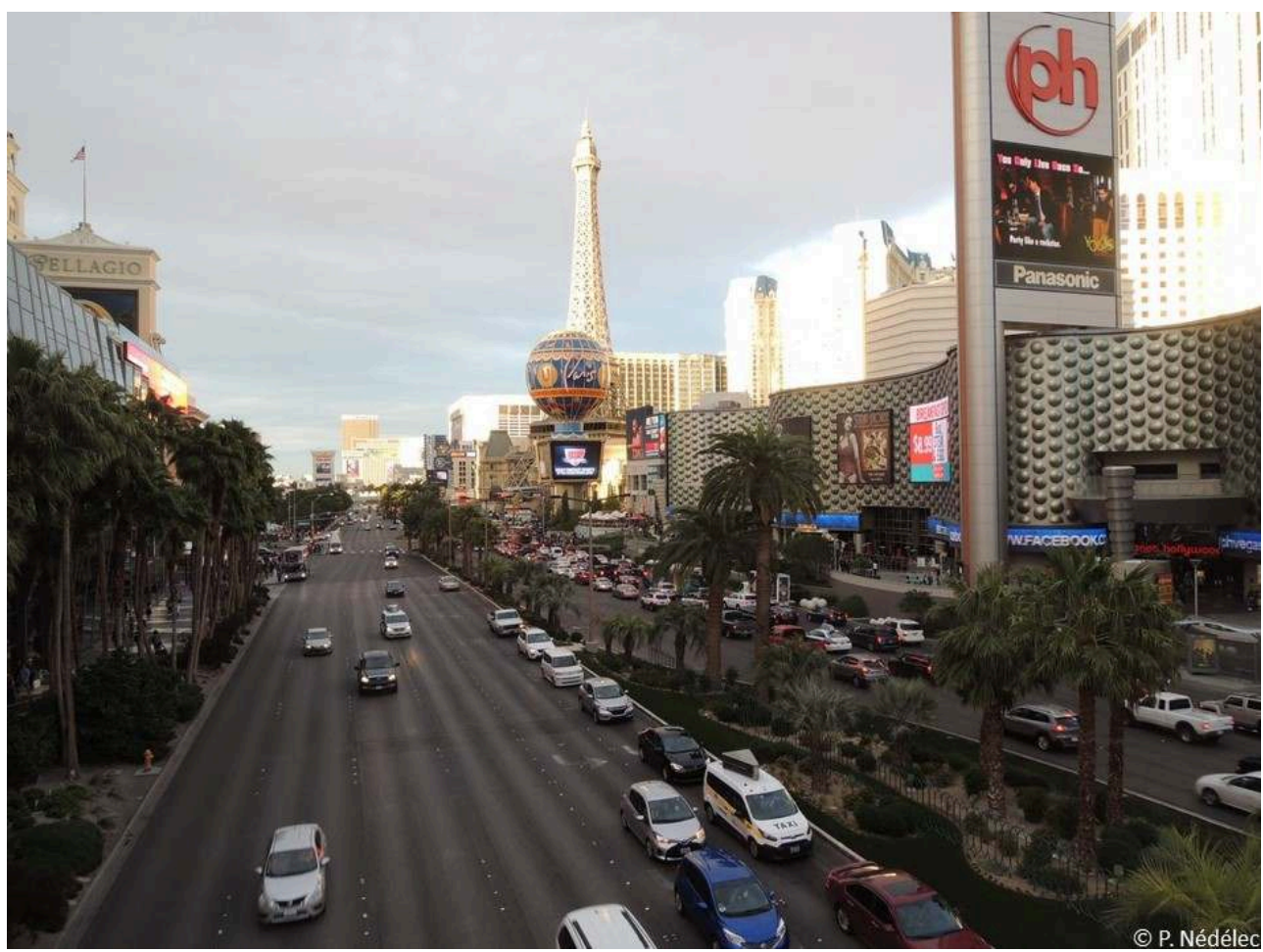

Vue depuis le pont piéton de Harmon Avenue, en direction du nord du Strip. A gauche de l'image, l'enseigne monumentale de l'hôtel-casino Planet Hollywood (PH) et la réplique de la Tour Eiffel de Paris, Las Vegas rappelle l'ancien calibrage autoroutier.

Source : P. Nédélec (2017)

Circuler en voiture le long du Strip est toujours une attraction recherchée, même si le boulevard est très fréquemment embouteillé. Les touristes qui déambulent en véhicules motorisés (taxis, voiture personnelle, bus publics ou cars touristiques) profitent ainsi d'une vue inégalée sur les architectures monumentales des établissements touristiques, encore plus impressionnantes la nuit lorsque les façades s'illuminent de milliers de jeux de lumière.

12 Le quartier touristique du Strip a ainsi été conçu pour et par des touristes automobilistes, de ses origines dans les années 1940 aux années 1980. A cette époque se produit un renouvellement majeur dans les modes d'appropriation du Strip : la marche à pied prend progressivement le pas sur les déplacements automobiles.

\section{L'affirmation de la marche comme nouvelle pratique touristique}

\section{L'aménagement des trottoirs par les hôtels-casinos du Strip : la « révolution Mirage»}

Si dans les années 1980 un grand nombre de visiteurs arrive toujours à Las Vegas en voiture, les déplacements automobiles perdent de l'importance lors des séjours végasiens au profit de déplacements pédestres le long du Strip. Cette bascule dans les modes de déplacement s'explique avant tout par l'aménagement des trottoirs par les 
hôtels-casinos qui en font de nouvelles extensions de leur offre touristique. Cette tendance est amorcée par l'hôtel-casino The Mirage, inauguré en 1989, entre Flamingo Road au Sud et Spring Mountain Road au Nord, soit le cœur géographique du Strip. Le Mirage, et son concepteur et propriétaire Steve Wynn, amorce un changement d'échelle qui établit le nouvel étalon pour les futurs établissements du Strip : le mega resort ou méga complexe hôtelier.

14 L'inauguration du Mirage est considérée comme un tournant fondamental dans la conception de l'offre touristique des hôtels-casinos végasiens à tel point que l'historien E. Moehring a parlé de « révolution Mirage » (Moehring E. et M. Green, 2005 : 210). Non seulement l'hôtel-casino dépasse en taille, en nombre de chambres (3 044, ce qui en faisait le plus grand hôtel du monde lors de son inauguration) et en élégance de la décoration tous ses concurrents, mais il ouvre aussi l'ère de l'omniprésence du divertissement avec une pléiade d'attractions aussi bien à l'intérieur qu'à l'extérieur du complexe hôtelier.

15 C'est ainsi dans l'extension de l'offre d'attractions sur les trottoirs devant l'établissement que réside l'originalité du Mirage. Pour la première fois, il n'est pas nécessaire de rentrer dans le bâtiment pour profiter des animations et attractions diverses. Homme visionnaire (Binkley C., 2008), Steve Wynn propose de faire des animations sur les trottoirs des produits d'appel destinés à donner envie aux visiteurs de pénétrer dans son hôtel-casino. Il construit pour cela un volcan artificiel (cf. photographies 2), marquant l'entrée du bâtiment et le séparant de la rue. Son emplacement est hautement stratégique puisqu'il est aussi bien visible depuis la rue, pour attirer les flux de piétons, que depuis la route, pour attirer l'œil des automobilistes, sans oublier depuis les chambres qui donnent sur le Strip.

Photographies 2 : Le Mirage et son volcan

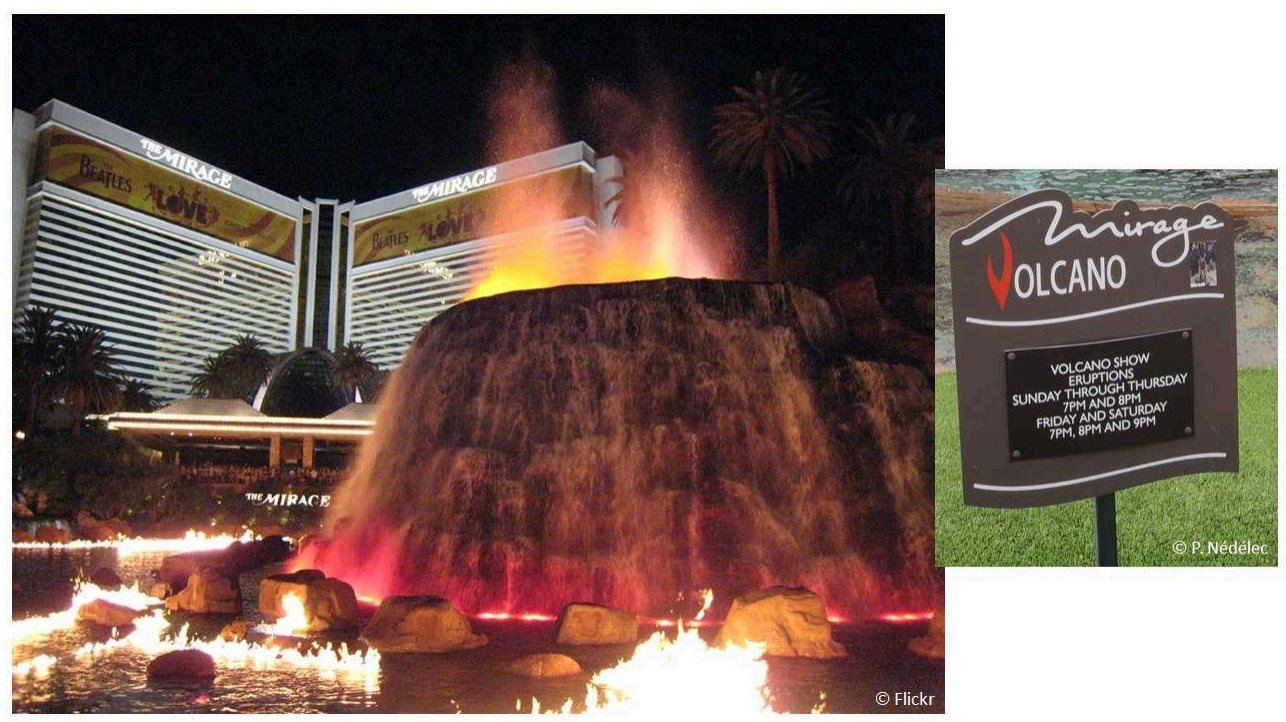

Sources : Ricky Lacy, Flickr (photographie de droite), P. Nédélec (photographie de gauche)

Le volcan est conçu comme un marqueur paysager du Mirage, dont il assure la promotion en en devenant l'image de marque. Outre son caractère paysager monumental (plus d'une dizaine de mètres de hauteur), le volcan devient une attraction plébiscitée lors de simulations d'éruptions volcaniques lorsque la nuit est 
tombée, qui engendrent des attroupements réguliers de touristes aux portes de l'établissement. Afin de faciliter les concentrations des touristes suscitée par cette attraction, la portion du trottoir devant le Mirage a même été élargie et des barrières ont été installées pour garantir à la fois la sécurité des badauds et leur confort pendant l'observation du spectacle (permettant ainsi de s'appuyer sur les rambardes).

L'aménagement des trottoirs constitue un affranchissement par rapport à la logique d'introversion qui présidait jusqu'alors. La conception des hôtels-casinos du Strip répondait en cela aux exigences de "directive élémentaire des casinos" (casino prime directive), qui consiste à garder le joueur / client à l'intérieur de son établissement (Nédélec P., 2012). Dans le sillage du Mirage et de son volcan, les trottoirs du Strip se parsèment dans la décennie 1990 d'attractions gratuites qui alimentent l'animation de la rue et en font un divertissement en soi : balades à gondoles devant The Venetian, spectacle son et lumière des fontaines du Bellagio, devenu l'une des attractions les plus recherchées du Strip, ou encore bataille pyrotechnique entre bateau pirate et sirènes devant l'hôtel-casino Treasure Island (cf. photographies 3, de gauche à droite).

Photographies 3 : Animations gratuites sur les trottoirs du Strip
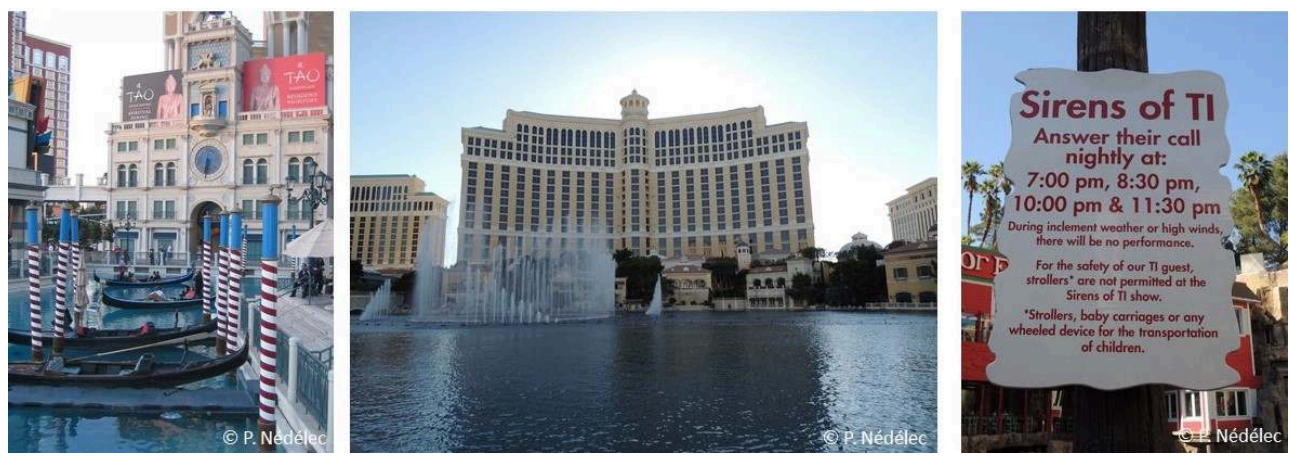

Source : P. Nédélec (2016)

Le deuxième apport révolutionnaire du Mirage consiste en l'ouverture d'une nouvelle ère architecturale, à savoir la généralisation de la thématisation architecturale des hôtels-casinos construits dans la décennie $1990^{6}$ (Nédélec P., 2016).

La multiplication des mega resorts thématiques, aux architectures monumentales et aux thèmes spectaculaires, ainsi que la multiplication d'attractions gratuites et accessibles à tous sur les trottoirs incitent dès lors un nombre grandissant de touristes à marcher le long du boulevard plutôt qu'à prendre leur voiture. En résulte une très rapide augmentation de la pratique piétonne qui donne une nouvelle image au Strip, digne des quartiers urbains les plus animés. La « révolution Mirage » et les choix audacieux des acteurs privés du secteur hôtelier sont ainsi les principaux promoteurs du renouvellement des pratiques de mobilité végasiennes.

\section{Un essor de la marche à pied accompagné par les pouvoirs publics}

Les acteurs publics végasiens ne sont toutefois pas passés à côté du renouvellement des pratiques touristiques. Bien au contraire, ils les ont accompagnées par des investissements publics dans l'aménagement du Las Vegas Boulevard et par l'offre de transports en commun. 
21 L'un des freins à la pratique de la marche avant les années 1990 résidait dans la difficulté pour un piéton de traverser l'ancienne autoroute intra-urbaine qu'est le Las Vegas Boulevard. En raison de sa taille, la traversée prend en effet plus de temps qu'une « simple » rue et l'ampleur du trafic routier accentuait les dangers pour les piétons. Les élus du comté de Clark, en charge de la voirie, ont pris conscience au début des années 1990 de l'impérieuse nécessité de repenser la voirie du Strip pour assurer la sécurité des piétons. Pour contourner les difficultés de traversée, un premier pont piétonnier surplombant la route est construit en 1994 à la jonction de Tropicana Avenue et du Las Vegas Boulevard. Cette intersection était particulièrement dangereuse pour les piétons puisqu'elle fait se croiser les deux axes routiers les plus empruntés de l'aire urbaine. Le principe d'un pont réservé aux seuls piétons et surplombant les voies routières a tout de suite convaincu les autorités locales, les propriétaires d'hôtels-casinos et les touristes. En effet, la construction a un impact limité sur la circulation, ne perturbant ainsi que peu le trafic routier dans son ensemble. De plus, cette solution présente l'avantage d'offrir des panoramas supplémentaires aux touristes pour profiter de points de vue sur les architectures grandiloquentes des hôtels-casinos du Strip. Depuis 1994, dix-sept autres ponts ont été construits le long du Strip, encadrant toutes les grandes intersections entre le Las Vegas Boulevard et les artères qui le traversent d'est en ouest. Le pont le plus récent a été inauguré en décembre 2019 et relie l'hôtel-casino Park MGM (anciennement Monte Carlo) et le centre commercial Showcase Mall. Il permet de surplomber le passage de soixante mille véhicules par jour et devrait être emprunté par une moyenne quotidienne de dix mille personnes selon les estimations du comté de Clark (Clark C., 2020). Cette infrastructure permet ainsi de faciliter l'accès au récent stade T-Mobile Arena (inauguré en avril 2016), augmentant d'autant son utilisation les jours d'évènements sportifs ou de spectacles culturels.

Parmi ces ponts, plusieurs débouchent directement dans les hôtels-casinos, renforçant le soutien des propriétaires pour qui les ponts piétonniers sont un outil supplémentaire de canalisation des flux en direction de leur établissement. Par exemple, les hôtelscasinos New York, New York et MGM Grand, qui se font face et appartiennent au même groupe MGM Resorts International, sont reliés l'un à l'autre par un pont surplombant. Les ponts sont doublés d'escalators qui permettent aux piétons de jouer avec les niveaux sans effort physique (cf. photographies 4).

Photographies 4 : Aménagements piétonniers de la voirie du Strip
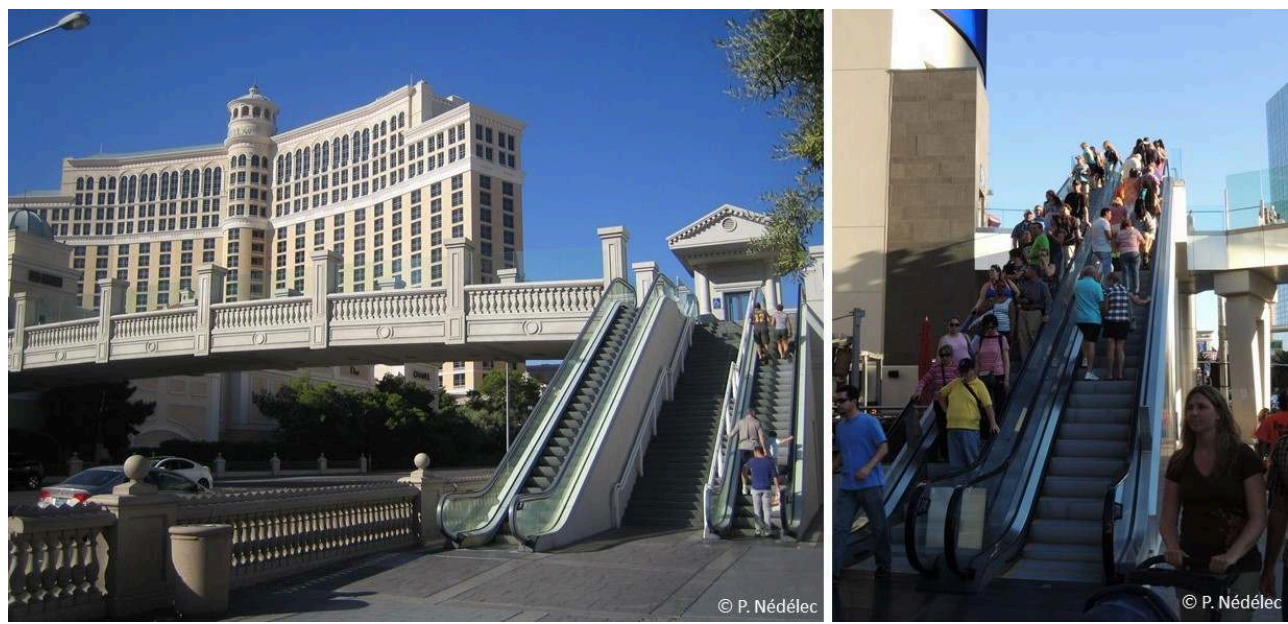

Source : P. Nédélec (2017) 
La ligne de bus à impériale « Deuce » constitue l'autre vecteur d'accompagnement par les pouvoirs publics locaux de l'essor de la marche à pied. Lancée en octobre 2005, la ligne de bus Deuce parcourt l'intégralité du Strip et en dessert les principaux hôtelscasinos qui donnent leur nom aux arrêts (cf. figure 2). Le nom même de la ligne fait référence à l'univers des jeux, puisqu'elle désigne un double chiffre lors d'un lancer de dés. La ligne est opérée par la branche végasienne de la régie de transports en commun du comté de Clark, la Regional Transportation Commission of Southern Nevada. Elle est financée en partie par les usagers et par des financements publics. Cet investissement fut un accélérateur incontestable de la pratique de la marche à pied le long du Strip. Sa popularité rapide et grandissante repose sur la proximité des arrêts. Grâce à elle, il n'est plus effrayant pour un touriste de se lancer dans une marche à pied puisqu'en cas de fatigue, le bus est là pour assurer la fin du parcours. Il faut ici rappeler que le Strip s'étire sur six kilomètres de long et que le climat estival peut être particulièrement harassant avec des températures moyennes supérieures à trente degrés entre mai et octobre, et même supérieures à quarante degrés entre juin et août.

Figure 2 : Desserte du Strip par la ligne de bus Deuce
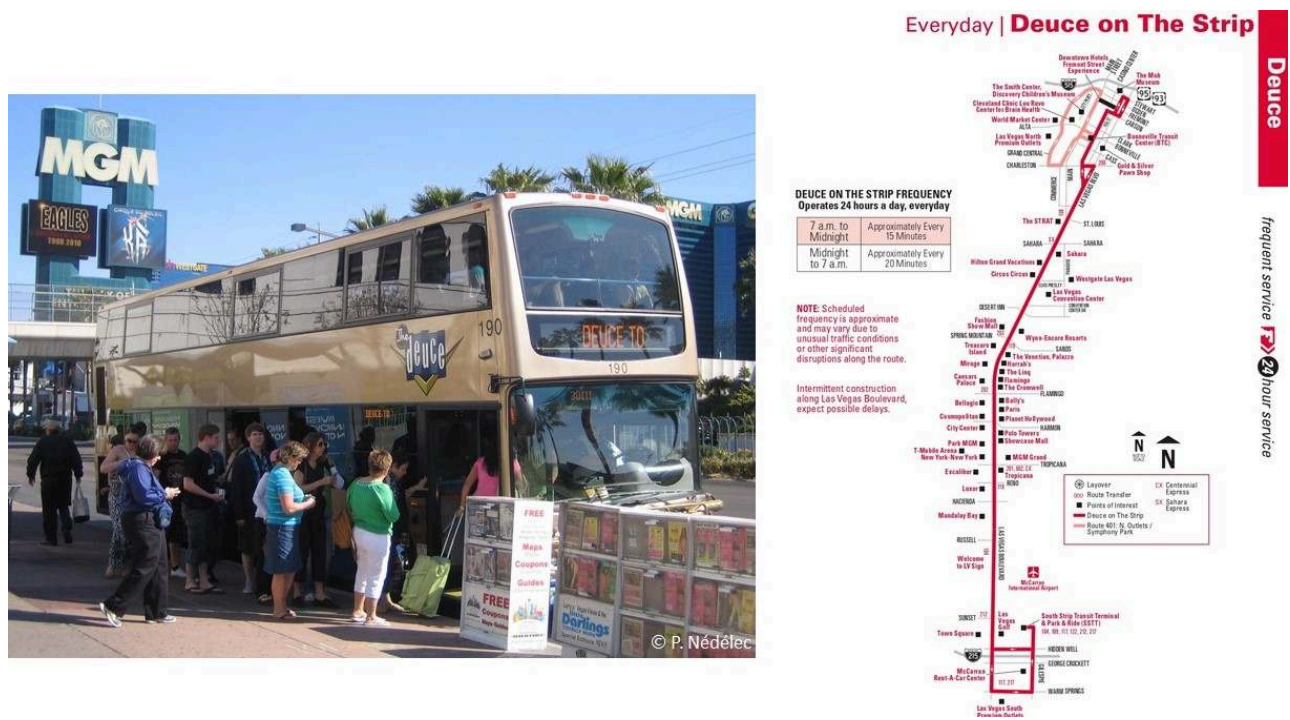

Sources : P. Nédélec (photographie de gauche), et Regional Transportation Commission of Southern Nevada

L'utilisation massive des transports en commun a conduit à l'installation de mobilier urbain aux principaux arrêts, signe distinctif de n'importe quelle grande ville. La présence de bancs peut paraître anodine mais elle incarne une autre "révolution" dans la conception et l'appropriation de l'espace public végasien. En effet, jusqu'à la fin des années 2000, aucun banc public n'était présent sur le Strip. Seuls les commerces étaient pourvus de tables et de chaises, conditionnant ainsi le fait de s'asseoir à une consommation payante. Ceci s'explique par la volonté des propriétaires d'hôtelscasinos de réduire toute concurrence, y compris pour la simple station assise. L'absence de banc et de chaise sur les trottoirs se comprenait ainsi d'autant mieux que l'entrée dans les hôtels-casinos est complètement gratuite et accessible à tous. Les touristes fatigués de marcher et cherchant à s'asseoir pouvaient le faire dans les établissements, devant une machine à sous ou devant une table de jeu, et ainsi en étant exposés à la tentation de jouer dans les casinos. 
L'affirmation de la marche à pied conduit à renouveler l'expérience touristique végasienne. La foule qui arpente les trottoirs fait vivre l'espace public de la rue et lui donne les apparences d'une ville vivante en pleine effervescence, donnant à voir le "hustle and bustle " caractéristique de la vie urbaine. L'appropriation du Strip par la déambulation piétonne éloigne les touristes de l'atmosphère confinée des casinos. La popularité de la marche à pied est telle que les trottoirs sont régulièrement encombrés et engendre de mini-embouteillages, particulièrement visibles aux passages piétons qui demeurent très usités malgré l'existence des ponts piétons (cf. photographies 5). Les trottoirs conçus à l'époque de la domination automobile s'avèrent aujourd'hui parfois inadéquats pour absorber l'intensité de la pratique piétonne. Des travaux réguliers sont entrepris par le comté de Clark pour adapter la voirie et les espaces de circulation aux piétons : élargissement de certaines portions du trottoirs, sécurisation des passages piétons, réfection des matériaux de surface, ajout de rambardes de protection, refonte de l'éclairage public, parterres végétalisés (Clark C., 2015).

Photographies 5 : Une appropriation piétonne du Strip digne des centres-villes les plus animés
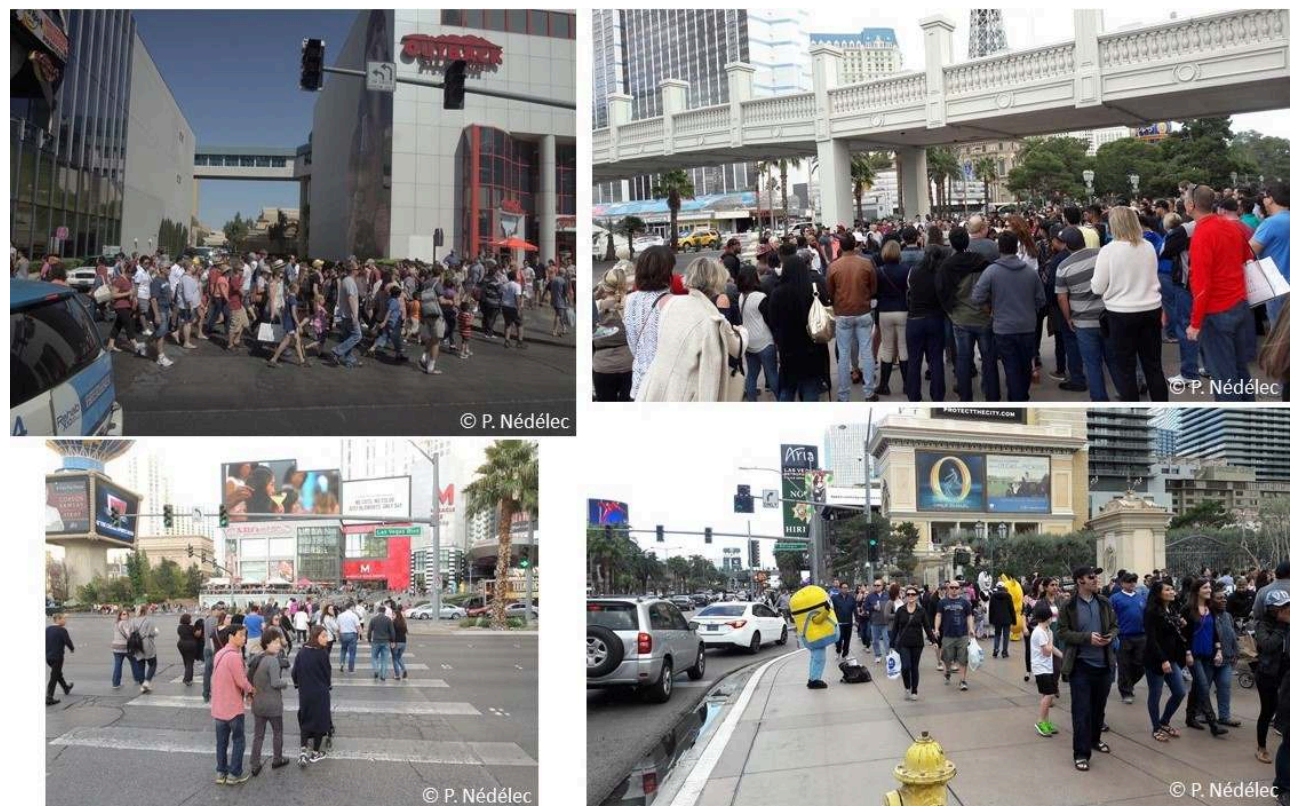

La popularité de la marche à pied le long du Strip est renforcée par la multiplication de divertissements temporaires, comme des spectacles de danse hip-hop (en haut à droite) ou la possibilité de se faire prendre en photo avec un personnage de film, ici un « Mignon » (en bas à droite). Source : P. Nédélec (2017)

\section{Marcher dans les rues plutôt que miser sur les tables : évolution structurelle des pratiques touristiques}

Devant l'attention croissante des médias comme des acteurs privés du secteur touristique envers la marche à pied, plusieurs rapports et enquêtes ont été réalisés à la fin des années 2000 pour quantifier les évolutions structurelles des pratiques touristiques. Ainsi, pour la première fois, l'ampleur des déplacements piétons est mesurée avec précision. En 2009, en moyenne environ dix-sept-mille-sept-cents personnes par heure marchent le long du Strip, quelle que soit l'heure ou le jour de la semaine. Des pics peuvent atteindre quarante-et-un-mille-deux-cents personnes par 
heure lors des périodes les animées de la saison touristique (grandes conventions ou spectacles populaires du printemps ou de l'automne). Dans le même rapport, il est estimé qu'en moyenne, un touriste marche 4,1 heures par jour le long du Strip, lui permettant de visiter 6,2 hôtels-casinos (Applied Analysis, 2009, Velotta R., 2009). De même, l'agence de promotion touristique locale LVCVA (Las Vegas Convention and Visitors Authority) a ajouté des questions sur les pratiques de mobilité quotidienne dans ses enquêtes annuelles auprès des touristes. Dans son rapport 2015, elle estime à $55 \%$ le nombre de touristes qui ont pratiqué la marche lors de leur séjour végasien, confirmant la popularité de cette pratique (LVCVA, $2015:$ 7). Ces chiffres n'ont fait qu'augmenter pour atteindre une moyenne journalière de cinquante-mille piétons en 2019 (Miller C., 2019).

L'appropriation de la marche à pied par une majorité de touristes en séjour à Las Vegas s'inscrit dans un mouvement plus large de diversification des activités touristiques proposées par les hôtels-casinos et autres établissements du Strip. Ceci a conduit à une réorganisation notable des postes de dépenses touristiques : depuis le début des années 2000 , les retombées financières issues de la pratique des jeux d'argent sont minoritaires. En 2011, les revenus des opérateurs d'hôtels-casinos végasiens n'étaient plus assurés qu'à 44,8 \% par les jeux d'argent alors qu'ils représentaient $61 \%$ en 1991 (LVCVA et Applied Analysis, 2012). Ceci témoigne de l'augmentation des dépenses des touristes pour d'autres types d'activités et pour l'attrait vers d'autres espaces que l'intérieur des casinos.

Ainsi, les dynamiques actorielles à l'œuvre dans l'affirmation de la marche à pied comme pratique touristique sont profondément imbriquées et fonctionnent de façon systémique. Amorcés par les acteurs du secteur touristique, les touristes se sont rapidement approprié les déplacements piétons grâce aux investissements publics, euxmêmes conçus comme un soutien à l'activité économique. La marche à pied est devenue la pierre angulaire de l'expérience touristique comme le résume la citation suivante, extraite d'un rapport des autorités du comté sur les aménagements nécessaires pour accompagner l'essor de la marche à pied: "La mobilité piétonne est centrale pour maintenir la vitalité économique et l'expérience du visiteur » (Clark C., 2015).

La référence à "l'expérience du visiteur » fait écho aux réflexions menées par les chercheurs sur le «tournant expérientiel » théorisé par les chercheurs en marketing (Frochot I. et W. Batat, 2013, Gombault A. et D. Bourgeon-Renault, 2014, Petr C., 2014). La notion de «tourisme expérientiel » s'inspire ainsi de ces travaux sur la prise en compte de l'expérience et des émotions individuelles dans les actes de consommation. Dans cette perspective, la volonté de s'adapter aux attentes des touristes en termes de voirie et de mobilier urbain (appropriés pour une pratique de la marche à pied) s'inscrit dans une logique plus large d'optimisation des incitations à la consommation, que ce soit dans les hôtels-casinos ou dans les centres commerciaux et autres commerces qui bordent le Strip. 


\section{Des reconfigurations spatiales portées par les hôtels- casinos pour accompagner le renouvellement des pratiques touristiques}

\section{L'engouement des acteurs du secteur touristique envers la marche à pied}

30 A première vue, la pratique de la marche à pied pourrait être combattue par les propriétaires d'hôtels-casinos et autres acteurs privés du secteur touristique car elle va à l'encontre de la «directive première » et de l'ambition d'une expérience touristique confinée aux limites d'un hôtel-casino ou d'un établissement touristique. Bien au contraire, ces acteurs ont pris conscience de l'évolution des mentalités et des attentes des touristiques et cherchent désormais à capitaliser sur ces nouvelles pratiques touristiques dominantes.

31 Depuis le milieu des années 2000, une nouvelle tendance s'observe ainsi sur le Strip : les acteurs du secteur touristique ont réagi face au désir croissant d'une interaction plus forte avec l'espace extérieur de la rue. Ce sont d'abord les propriétaires de bars et de restaurants qui ont joué la carte de l'ouverture et le nombre d'établissements ouverts sur l'extérieur s'est multiplié : les terrasses et les balcons ont fleuri et sont autant d'occasions pour les clients attablés aux tables de profiter du spectacle de la rue. Parmi les activités plébiscitées dans ces nouveaux espaces extérieurs, l'observation des passants (people watching) se dispute avec l'affichage ostentatoire des marques d'une soirée réussie, comme de nombreuses bouteilles d'alcool à sa table accompagnée de beaux jeunes gens vêtus à la dernière mode.

Même si ces terrasses sont encore essentiellement incluses dans les hôtels-casinos ou les centres commerciaux, donc dans des espaces de droit privé qui ont vue sur l'espace public de la rue, elles redéfinissent l'expérience même du Strip le long duquel la séparation stricte entre l'intérieur des hôtels-casinos et la rue s'affaiblit de plus en plus. L'année 2013 représente une nouvelle étape de rupture forte dans la conception des établissements touristiques et dans l'affranchissement par rapport à une architecture introvertie et conçue en vase clos. Portés par les groupes hôteliers MGM Resorts International et Caesars Entertainment, débute la construction de deux projets urbains entendant parer le Strip des attributs urbains dignes des plus grandes villes du monde, à savoir des places publiques et rues piétonnes (Ghorra-Gobin C., 2001).

\section{"The Linq Promenade " et "The Park Vegas » : reconfigurations spatiales et nouveau visage du Strip}

Deux projets urbains incarnent l'inversion complète du paradigme des hôtels-casinos opérée au début du $\mathrm{XXI}^{\mathrm{e}}$ siècle afin de répondre au renouvellement des pratiques touristiques de mobilité. La rue piétonne « The Linq Promenade » et la place « The Park Vegas» illustrent les reconfigurations spatiales qui découlent de l'affirmation de la marche à pied. Parce que ces aménagements créent de nouveaux espaces publics - tout du moins d'apparence -, ils incarnent une rupture complète avec la conception dominante jusque là d'hôtels-casinos tournant le dos à la rue et à l'extérieur. Le tableau 1 présente de façon synthétique ces deux aménagements. 
Tableau 1 : Synthèse des aménagements urbains The Linq Promenade et The Park Vegas

\begin{tabular}{|l|l|l|}
\hline & The Linq Promenade & The Park Vegas \\
\hline Localisation & $\begin{array}{l}\text { Entre les hôtels-casinos the } \\
\text { Linq et Flamingo }\end{array}$ & $\begin{array}{l}\text { Entre les hôtels-casinos New York New York and } \\
\text { Park MGM (anciennement Monte Carlo) }\end{array}$ \\
\hline Coût & 550 millions de dollars & 100 millions de dollars \\
\hline Inauguration & Mars 2014 & Avril 2016 \\
\hline Superficie & $\begin{array}{l}\text { Rue piétonne de 380 mètres } \\
\text { de long }\end{array}$ & 1,2 hectare \\
\hline $\begin{array}{l}\text { Promoteur } \\
\text { Architecte) }\end{array}$ & $\begin{array}{l}\text { Caesars Entertainment } \\
\text { (David M. Schwarz) }\end{array}$ & $\begin{array}{l}\text { MGM Resorts International } \\
\text { (Cooper, Robertson \& Partners) }\end{array}$ \\
\hline $\begin{array}{l}\text { Principale } \\
\text { attraction }\end{array}$ & $\begin{array}{l}\text { Grande roue « High Roller » } \\
\text { (167 m.) }\end{array}$ & $\begin{array}{l}\text { Stade T-Mobile Arena } \\
\text { (20 000 places) }\end{array}$ \\
\hline $\begin{array}{l}\text { Références } \\
\text { urbaines }\end{array}$ & $\begin{array}{l}\text { York } \\
\text { Bourbon Street, New Orleans }\end{array}$ & \begin{tabular}{l} 
Madison Square Park, New York \\
\hline
\end{tabular} \\
\hline
\end{tabular}

Source : P. Nédélec (2020)

Le principe sous-tendant ces deux réalisations est identique : créer un environnement typiquement urbain, incitant à la flânerie et aux interactions sociales. C'est New York, et plus précisément Manhattan, qui aurait inspiré les deux opérateurs, The Linq Promenade cherchant à imiter le quartier du Meatpacking District et The Park Vegas la place du Madison Square (Peterson K., 2013). La référence new yorkaise est loin d'être anodine dans le plan de communication des deux opérateurs : ils se revendiquent ainsi dans la lignée d'une ville qui est considérée dans les représentations collectives étatsuniennes comme l'incarnation par excellence de l'animation urbaine. En voulant reproduire des morceaux de Manhattan le long du Strip, les acteurs du tourisme cherchent à développer ces pratiques urbaines typiques des grandes villes, comme la marche, et ainsi faire taire les critiques qui reprochent au Strip son manque d'authenticité urbaine (Bégout B., 2002, Nédélec P., 2017 : 52-62). Le choix de l'agence d'architectes Cooper, Robertson \& Partners pour la conception du Park Vegas est en cela significatif puisqu'elle s'est fait remarquer par ses réalisations d'espaces urbains emblématiques, tels que l'esplanade de Battery Park City et le parc Zuccotti à New York, ou la réhabilitation du front de mer de Cityfront Center à Chicago (Stutz H., 2013). Le PDG de MGM Resorts, Jim Murren, affiche ainsi clairement ses ambitions de rivaliser avec n'importe quel autre quartier d'une grande ville :

Les consommateurs d'aujourd'hui ne trouvent plus aussi attirant l'ancien concept [de casinos coupés de la rue et tournés sur eux-mêmes]. Notre vision est de changer de façon spectaculaire le trottoir le long du Las Vegas Strip, en créant un espace qui améliorera grandement la zone et qui créera une énergie que vous trouvez dans les plus grandes villes du monde. [...] Un visiteur de Las Vegas ne veut plus être 
enchaîné à un complexe touristique. Ce même visiteur veut se déplacer et être social. C'est ce que ce type d'aménagement offre aux clients.

Stutz H., 2013 considérée comme classique, organisée autour des espaces publics. En cela le changement est net avec les répliques en carton-pâte d'environnements urbains confinés à l'intérieur des casinos où l'on reproduit en faux jusqu'au ciel bleu, comme c'est le cas par exemple dans le Paris Las Vegas. Indéniablement, il s'agit ici d'espaces extérieurs structurés par des allées accueillantes, une végétalisation et un mobilier urbain conséquent pour créer un environnement urbain chaleureux (cf. photographie 6a). Le recours important aux plantations locales et aux plans d'eau constitue une autre nouveauté notable. La végétation relève du xeriscape, c'est-à-dire un paysage composé de végétaux résistants à la chaleur et originaires du désert de Mojave. Fournir de l'ombre et de la fraîcheur est essentiel pour rendre la place praticable pendant la saison estivale, la chaleur accablante ayant longtemps été le principal argument censé décourager les touristes de s'aventurer et de marcher à l'extérieur des hôtels-casinos ou des centres commerciaux climatisés.

Photographies 6a : Des espaces d'apparence publique...
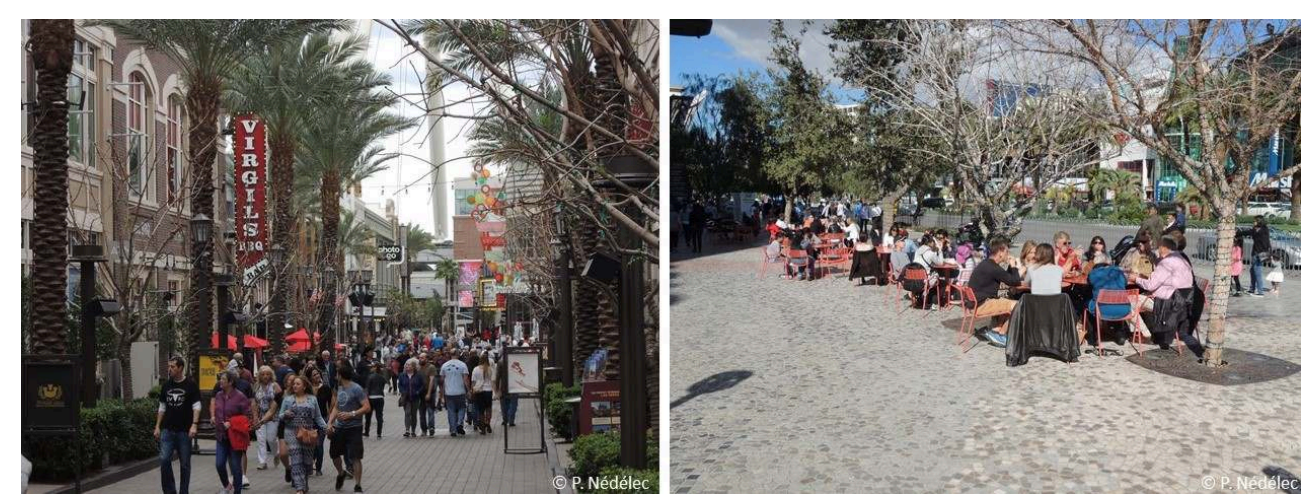

A gauche, The Linq Promenade ressemble à une rue piétonne animée, bordée de commerces. A droite, The Park Vegas ressemble à une place publique, parsemée de tables et de bancs accessibles à tous (sans nécessité de consommer).

Source : P. Nédélec (2017)

La valorisation de l'espace public et de la liberté de circulation n'efface pour autant pas les objectifs commerciaux : c'est bien aux «clients» des casinos que s'adressent ces aménagements et la rentabilité économique prédomine. La communication de Caesars Entertainment est ainsi plus explicite quant à ses objectifs commerciaux puisqu'elle décrit The Linq Promenade comme un «quartier de commerces en plein air, de restaurants et de loisirs». The Linq Promenade joue ainsi plus la carte du 
divertissement que de l'expérience urbaine : le projet s'articule en effet autour d'une immense grande roue, surnommée "High Roller», littéralement le flambeur, en hommage aux riches habitués des tables de jeu. Haute de 167 mètres et d'un diamètre de 158 mètres, elle a pour ambition « d'éclipser à la fois la London Eye et le Singapore Flyer ». Derrière la communication, la conception de ces deux aménagements urbains se rapproche ainsi plus de quartiers ludiques axés sur la consommation (Gravari-Barbas M., 2000) que d'espaces publics urbains au sens plein du terme (Tonnelat S., 2016).

\section{Des aménagements urbains entre publicisation apparente et privatisation de fait}

The Linq Promenade comme The Park Vegas n'échappent pas à la volonté de contrôle total activement recherché par les hôtels-casinos au travers d'une privatisation des trottoirs (Nédélec, P., 2012). En dépit des apparences, ces deux aménagements sont des espaces de droit privé dans lesquels les usages sont fortement régulés et réglementés, avec pour principal objectif de faciliter les flâneries consuméristes. Les bancs et les tables ne sont certes pas réservés aux consommateurs, ce qui est rare sur le Strip comme on l'a décrit plus haut, mais les agents de sécurité privée qui surveillent ces espaces sont autorisés à chasser toute personne ne respectant pas les règles des hôtelscasinos propriétaires des lieux.

Photographies $6 \mathrm{~b}:$... mais des espaces privés contrôlés par les hôtels-casinos
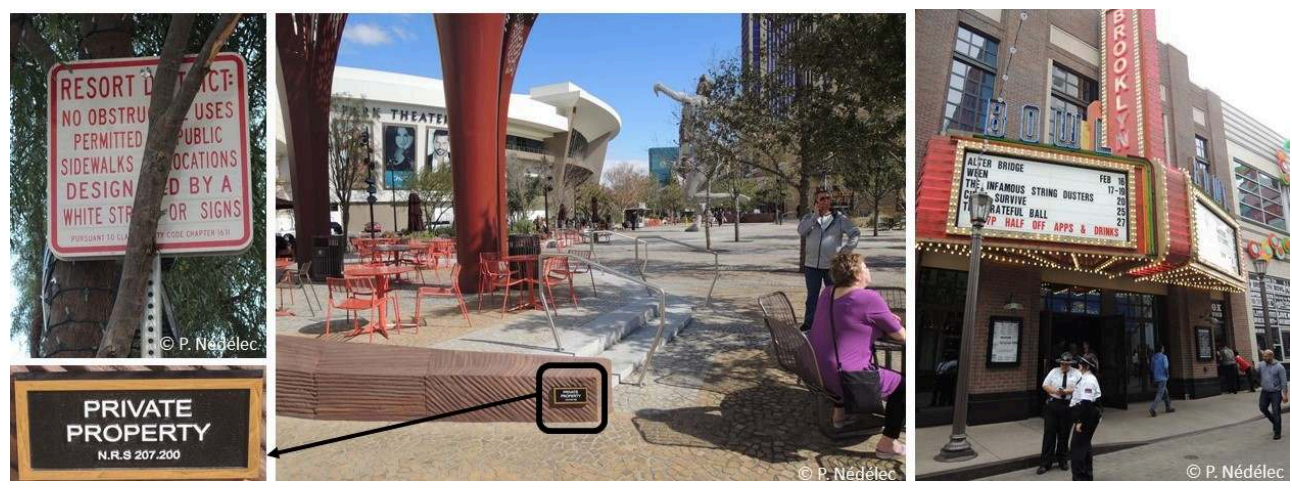

A gauche, des panneaux peu visibles du grand public non averti signale le caractère privé du Park Vegas. A droite, des vigiles de sécurité surveillent au nom des opérateurs privés la bonne tenue des usagers du Linq Promenade.

Source : P. Nédélec (2017)

Les indices dissimulés dans la végétation ou dans les angles morts des touristes témoignent de la privatisation de ces espaces d'apparence publique. La Linq Promenade est ainsi soumise au contrôle d'agents de sécurité privée, dont la tenue ressemble à celle d'une police locale, comme l'indique le badge sur le chapeau de l'agent (cf. photographies $6 \mathrm{~b}$, à droite). Les marqueurs du caractère privé de Park Plaza sont encore plus allusifs : une plaque, cachée sur une bordure des plates-bandes, déclare le statut d'"espace privé» (private property) au nom de la «N.R.S 207.200» (cf. photographies $6 \mathrm{~b}$, à gauche). Cet acronyme fait référence à la législation de l'Etat du Nevada (Nevada Revised Statutes), et plus spécifiquement au chapitre 207 qui définit la criminalité, et à son article 200 qui définit la violation de propriété privée. Ainsi, c'est bien le groupe MGM Resorts International, propriétaire des deux hôtels-casinos 
bordant la place qui décide des usages autorisés ou non, indépendamment de leur (il)légalité. Des usages parfaitement légaux peuvent ainsi être interdits par le propriétaire comme les manifestations et autres rassemblements politiques (Nédélec P., 2012).

Ces lieux sont ainsi des mises en scène d'espaces publics, aménagés pour répondre au renouvellement des pratiques touristiques tout en maintenant la mainmise des acteurs privés sur les usages et les comportements autorisés dans leurs propriétés. Si la reconfiguration spatiale est nette et incontestable, sa publicisation est factice et révèle l'ampleur des logiques commerciales au fondement de la conception de l'urbanité végasienne.

\section{Mise en relation des évolutions du Strip et du reste de l'aire urbaine végasienne}

41 En changeant d'échelle d'analyse, les évolutions du quartier touristique du Strip peuvent être mises en relation avec les dynamiques de l'ensemble de l'aire urbaine végasienne. La popularité grandissante de la marche à pied parmi les touristes contraste très fortement avec les pratiques de mobilité dominantes parmi les deux millions de Végasiens. En effet, en dehors des quartiers touristiques, ce sont toujours les mobilités automobiles qui dominent dans une aire urbaine conçue à partir des années 1910 autour de la voiture. On constate ainsi un décalage soutenu entre le renouvellement des pratiques des touristes et celles des habitants.

Les évolutions en revanche sont plus similaires lorsque l'on s'intéresse à la volonté des acteurs locaux de renouveler les représentations collectives associées à Las Vegas. Les projets urbains de The Park Vegas et The Linq Promenade participent en effet de la mue de l'aire urbaine végasienne qui se dote progressivement des attributs urbains qui lui faisaient encore défaut pour prétendre au statut de "vraie ville» aux yeux des Etats-Uniens : un stade pour accueillir une équipe sportive de rayonnement national ${ }^{7}$ (équipe de football américain The Raiders) et le renouveau du downtown (centre-ville de City of Las Vegas) de plus en plus vivant et «branché $»^{8}$. Ces initiatives locales s'inscrivent dans un mouvement de fond depuis les années 2000 qui cherche à déconstruire les représentations partielles et partiales construites à partir du seul quartier touristique du Strip, qui masquent la «normalisation» de Las Vegas (Gottdiener M. et al., 1999). Les acteurs locaux ont ainsi à cœur de faire prendre conscience aux Etats-Uniens que l'aire urbaine végasienne est diversifiée fonctionnellement et économiquement, offrant ainsi de nombreuses possibilités professionnelles et éducatives ${ }^{9}$, et qu'elle offre un cadre de vie privilégié caractérisé par de relativement faibles coûts de l'immobilier (notamment par rapport au Sud de la Californie) et un climat très agréable.

\section{Conclusion}

En conclusion, le renouveau de la désirabilité sociale de la marche à pied illustre le rôle des pratiques culturelles dans la fabrique matérielle de la ville et ses reconfigurations. L'exemple des évolutions du quartier touristique du Strip entre les années 1990 et 2010 démontre que des pratiques culturelles aussi simples et banales que la marche à pied peuvent engendrer une inversion complète du paradigme spatial qui régissait 
l'organisation d'acteurs privés aussi conséquents que les propriétaires d'hôtels-casinos. Cet exemple illustre également l'imbrication actorielle entre acteurs privés du secteur touristique, acteurs publics locaux et acteurs individuels dans la promotion d'une pratique de mobilité piétonne le plus souvent pensée à l'échelle individuelle. Dès lors, marcher dans les rues de Las Vegas repose autant sur une évolution collective des pratiques culturelles que sur un repositionnement marketing d'acteurs privés qui misent sur les retombées financières de l'essor de la marche à pied, le tout soutenu par des aménagements de la voirie et par un urbanisme pro-piéton mis en place par les autorités locales.

The Linq Promenade comme The Park Vegas sont des reconfigurations spatiales qui participent non seulement de l'ouverture sur l'extérieur et de la reconquête de l'espace de la rue, mais aussi d'un renouveau des représentations de Las Vegas dans l'opinion collective états-unienne, fréquemment accusée de ne pas être une "vraie ville" (Nédélec P., 2017). Bien que la privatisation des espaces ouverts domine encore, ces projets urbains permettent néanmoins de changer le récit végasien et participent de sa revendication de légitimité urbaine.

\section{BIBLIOGRAPHIE}

Bégout, Bruce, Zéropolis : l'expérience de Las Vegas, Paris, Allia, 2002

Binkley, Christina, Winner Takes All: Steve Wynn, Kirk Kerkorian, Gary Loveman, and the Race to Own Las Vegas, New York, Hyperion, 2008.

Clark County, Pedestrian Study: Las Vegas Boulevard [en ligne], 2015, https:// www.clarkcountynv.gov/government/departments/public_works_department/ programs_and_services/las_vegas_blvd.php, page consultée le 27 janvier 2021.

Clark County, « New Pedestrian Bridge Opens Over Las Vegas Strip » [en ligne], 3 février 2020, https://youtu.be/6NcVyyAeCJc, page consultée le 27 janvier 2021.

Cooper Robertson, « The Park and Las Vegas Boulevard Streetscape » [en ligne], 2015, https:// www.cooperrobertson.com/work/the_park_and_las_vegas_boulevard_streetscape, page consultée le 27 janvier 2021.

Frochot, Isabelle et Batat, Wided, Marketing and Designing the Tourist Experience, Goodfellow Publishers Limited, 2013.

Ghorra-Gobin, Cynthia (dir.), Réinventer le sens de la ville : les espaces publics à l'heure globale, Paris/Montréal, L'Harmattan, 2001.

Gombault, Anne et Bourgeon-Renault, Dominique, « Marketing du tourisme. Le tournant expérientiel », Revue Espaces, n³20, 2014.

Gottdiener, M. et al., Las Vegas, The Social Production of an All-American City, Oxford, Blackwell, 1999. Gravari-Barbas, Maria, La ville festive. Espaces, expressions, acteurs, Habilitation à diriger des recherches, Université d'Angers, 2000. 
LVCVA, Las Vegas Visitors Profile 2015, 2015.

LVCVA et Applied Analysis, Destination Diversification: Non-gaming vs. Gaming impacts in Las Vegas [en ligne], septembre 2012, http://www.appliedanalysis.com/projects/lvcvaeis/LVCVAEIS-0912.pdf, page consultée le 27 janvier 2021.

Miller, Cody, « Latest pedestrian bridge over the Strip to open before Christmas » [en ligne], KSNV, 22 décembre 2019, https://news3lv.com/news/local/latest-pedestrian-bridge-over-thestrip-to-open-before-christmas, page consultée le 27 janvier 2021.

Moehring, Eugene, Resort City in the Sun Belt: Las Vegas 1930-2000, Reno, University of Nevada Press, 2000.

Moehring, Eugene et Green, Michael, Las Vegas, A Centennial History, Reno, University of Nevada Press, 2005.

Nédélec, Pascale, « L'enclave fonctionnelle du Strip à Las Vegas : quand l'insularité façonne la ville », Espaces et Sociétés, n¹50, 2012, p. 49-65.

Nédélec, Pascale, « Quand spécialisation touristique rime avec bling-bling : Las Vegas, destination ostentatoire? », Urbanités [En ligne], juin 2016, https://www.revue-urbanites.fr/7-quandspecialisation-touristique-rime-avec-bling-bling-las-vegas-destination-ostentatoire/, page consultée le 27 janvier 2021.

Nédélec, Pascale, «Conséquences du renouvellement de la gouvernance urbaine dans le centreville de City of Las Vegas : l'action de Tony Hsieh et du Downtown Project », L'Ordinaire des Amériques [En ligne], novembre 2016, https://journals.openedition.org/orda/3051, page consultée le 27 janvier 2021.

Nédélec, Pascale, Las Vegas dans l'ombre des casinos. (Dé)construire l'urbanité et la citadinité végasiennes, Rennes, Presses Universitaires de Rennes, 2017.

Peterson, Kristen, « Outside In? », Las Vegas Weekly, 15- avril - 1er mai 2013, p.10.

Petr, Christine, « Le tourisme expérientiel, ça n'existe pas... mais c'est utile à la réflexion marketing », Revue Espaces, n³20, p.34-39, 2014.

Schwartz, David, Suburban Xanadu: the Casino Resort on the Las Vegas Strip and Beyond, New York, Routledge, 2003.

Stutz, Howard, « MGM has grand plans with trendy plaza, sports arena », Las Vegas Review Journal, 18 avril 2013, https://www.reviewjournal.com/business/casinos-gaming/mgm-has-grand-planswith-trendy-plaza-sports-arena/, page consultee le 27 janvier 2021.

Tonnelat, Stéphane, « Espace public, urbanité et démocratie », La Vie des idées [En ligne], mars 2016, https://laviedesidees.fr/Espace-public-urbanite-et-democratie.html, page consultée le 27 janvier 2021.

Velotta, Richard, « How many walk LV Strip? Mobile message deliverer now knows the answer », Las Vegas Sun, 26 juin 2009, https://lasvegassun.com/news/2009/jun/26/how-many-walk-lvstrip-mobile-message-deliverer-no/, page consultée le 27 janvier 2021.

Venturi, Robert, Scoot Brown, Denise, et Izenour, Steven, Learning from Las Vegas, Cambridge, MIT Press, 1972.

Venturi, Robert, Scoot Brown, Denise, et Izenour, Steven, Learning from Las Vegas: The Forgotten Symbolism of Architectural Form. (Revised edition), Cambridge, MIT Press, 1977. 


\section{NOTES}

1. L'aire urbaine de Las Vegas s'étend sur le territoire de trois municipalités distinctes (City of Las Vegas, North Las Vegas et Henderson) ainsi qu'une partie du territoire non-incorporé du comté de Clark.

2. L'Etat du Nevada détient le monopole de la pratique légale des jeux d'argent aux Etats-Unis jusqu'en 1978 et l'autorisation des jeux d'argent dans l'Etat du New Jersey. Depuis 1978, la plupart des Etats fédérés autorise une forme ou une autre de jeux d'argent, confirmant la démocratisation et la banalisation de cette pratique récréative.

3. Last Frontier (1943), Flamingo (1946), Desert Inn (1950), The Sands et Sahara (1952), Riviera (1955), Dunes (1955), Hacienda (1956), Tropicana (1957), Stardust (1958), Imperial Palace (1959).

4. Le Las Vegas Strip serait une référence au Sunset Strip, la portion la plus commerçante du Sunset Boulevard à Los Angeles.

5. C'est la rue de Fremont Street, dans le centre historique de la municipalité de City of Las Vegas, qui a concentré les premiers hôtels-casinos végasiens à partir des années 1900.

6. The Excalibur (1990), le second MGM Grand (1993), Treasure Island (1993), New York, New York (1997), Luxor (1993), The Venetian (1999), et Paris Las Vegas (1999).

7. L'Allegiant Stadium, inauguré en juillet 2020, accueille des matchs des championnats de football américain de la NFL (National Football League) et de la NCAA (National Collegiate Athletic Association).

8. Le renouveau du centre-ville historique de City of Las Vegas est étroitement associé aux investissements de l'homme d'affaires Tony Hsieh (Nédélec P., 2016).

9. L'aire urbaine compte une université d'Etat (University of Las Vegas, Nevada - UNLV) et un « community college» (College of Southern Nevada-CSN).

\section{RÉSUMÉS}

Cet article étudie l'essor de la marche à pied dans le quartier touristique du Strip à Las Vegas depuis les années 1990. L'affirmation de la marche à pied comme principale pratique touristique constitue une rupture nette aussi bien dans les pratiques de mobilité dominantes que dans l'organisation physique du Strip. Le quartier a en effet été conçu dans les années 1940 autour des déplacements automobiles. Cet article étudie plus particulièrement la dimension actorielle de l'évolution des pratiques touristiques de mobilité en interrogeant le rôle respectif des acteurs privés propriétaires des hôtels-casinos, des acteurs publics et des acteurs individuels que sont les touristes. Les reconfigurations spatiales engendrées par l'essor de la marche à pied sont étudiées en détail à partir de deux aménagements touristiques récents (années 2010) : the Linq Promenade et The Park Vegas. Ces derniers confirment la rupture dans la conception même des hôtelscasinos du Strip : de l'introversion vers l'extraversion et l'extension dans le domaine public de la rue et des trottoirs. Ils sont l'occasion de mettre en regard une pratique piétonne ancrée dans les espaces publics et la privatisation effective de ces lieux touristiques.

This paper studies the rise of walking on the Strip, Las Vegas main tourist neighborhood, since the 1990s. Walking has flourished as a major tourist practice, embodying a distinct breach not only in the way tourist move around but also in the lay-out of the Strip. This tourist neighborhood has indeed been conceptualized and organized by and for car drivers since its 
origins in the 1940s. This paper studies more precisely the local power plays underlying the evolution of tourist mobility practices, thus analysis successively the role of hotel-casinos operators, local officials, and tourists. The rise of walking has led to spatial reconfigurations both throughout the Strip and at the level of some hotel-casinos. Two recent developments are examined more thoroughly: the Linq Promenade and the Park Vegas, both built in the 2010s. They are deemed exemplary of the new extraversion of hotel-casinos to fit the renewed walking expectations of tourists. They are also interesting to highlight the tension between seemingly open spaces and their de facto privatization.

Este artículo estudia el desarrollo de la caminata en el barrio turístico del Strip en Las Vegas desde la década de los 90. La confirmación de la caminata como práctica turística principal constituye una clara ruptura tanto en las prácticas de movilidad dominantes como en la organización física del Strip. De hecho, el barrio fue diseñado en los años 1940 en torno a los desplazamientos en automóvil. Este artículo estudia más específicamente los juegos locales de poder en la evolución de las prácticas de turismo de movilidad, al cuestionar los papeles respectivos de los actores privados, dueños de hoteles casino, de los actores públicos y de los actores individuales como los turistas. Las reconfiguraciones espaciales generadas por el auge de la caminata se estudian en detalle a partir de dos proyectos turísticos recientes (década de 2010): the Linq Promenade y The Park Vegas. Estos confirman la ruptura en la concepción misma de los hoteles-casino del Strip: de la introversión a la extroversión y la extensión al ámbito público de las calles y aceras. Son una oportunidad para comparar una práctica peatonal arraigada en los espacios públicos y la privatización efectiva de estos lugares turísticos.

\section{INDEX}

Mots-clés : Marche à pied, pratiques touristiques, reconfigurations spatiales, projet urbain, Las Vegas

Palabras claves : caminar, prácticas turísticas, reconfiguraciones espaciales, planificación urbana, Las Vegas

Keywords : walking, tourist practices, spatial reconfigurations, city planning, Las Vegas

\section{AUTEUR}

\section{PASCALE NÉDÉLEC}

Pascale Nédélec est docteure agrégée en géographie, enseignante en CPGE au lycée Janson de Sailly à Paris. Ses recherches portent sur la géographie urbaine, notamment aux Etats-Unis. Elle a plus particulièrement étudié la spécialisation touristique de l'aire urbaine de Las Vegas et ses conséquences sur la population locale. Parmi ses principales publications : Atlas des Etats-Unis (2021) aux éditions Autrement, manuel de Géographie urbaine (2018) chez Colin et Las Vegas dans l'ombre des casinos aux Presses Universitaires de Rennes (2017). 